\title{
Erosion patterns on a granular bed around a vertical cylinder
}

\author{
Florent Lachaussée ${ }^{1}$, Yann Bertho ${ }^{1}$, Cyprien Morize ${ }^{1}$, Alban Sauret ${ }^{1,2}$, and Philippe Gondret ${ }^{1, \star}$ \\ ${ }^{1}$ Laboratoire FAST, Univ. Paris-Sud, CNRS, Université Paris-Saclay, F-91405, Orsay, France \\ ${ }^{2}$ Laboratoire SVI, CNRS, Saint-Gobain, 39 quai Lucien Lefranc, F-93303 Aubervilliers, France
}

\begin{abstract}
We report on two different patterns that can be observed at the bed surface close to a vertical cylinder when submitted to a strong enough steady water flow. The classical scour pattern observed at the cylinder foot and due to the "horseshoe" vortex around occurs at a critical velocity $U_{c_{1}}$ below the critical velocity $U_{c_{0}}$ for erosion without any cylinder, thus under clear-water conditions. But we observe also another pattern downstream the cylinder which consists of two symmetrical ovoid holes that look like "bunny ears". This new scour pattern referred as BES can be observed at lower velocities that the horseshoe scour (HSS), above a critical velocity $U_{c_{2}}<U_{c_{1}}$, with a timescale formation much higher that the one of HSS.
\end{abstract}

\section{Introduction}

Erosion is encountered in numerous natural or industrial situations. Understanding erosion process is essential for instance to interpret geomorphological patterns on Earth or other planets and to predict the evolution of estuaries and river beds [1]. But quantifying the erosion processes generated at the vicinity of structures referred as scouring is also relevant to a wide range of engineering applications, including the design and risk assessment of hydraulic structures such as bridge piers, off-shore platforms and wind turbines. There has been a huge amount of research carried out to address this question with large scale facilities [2], but the physics of local scour phenomena occurring around structures is far from being understood [3]. This complexity arises both from the complex flow around a cylinder [4] and the complex rheology of granular material [1]. Numerical approaches are far from describing carefully these two complexity levels and also the complex grain/fluid interaction even if key advances have been made with the recent development of coupled methods with Large Eddy Simulation (LES) for the fluid and Discrete Element Method (DEM) for the grain phase [5].

In the present paper, we focus on the simplified case of scouring at the base of a cylindrical structure of circular cross-section. This geometry represents the traditional template for studying scour processes around structures and finds important applications in civil and off-shore engineering. We present experimental results from a smallscale setup that allows easy monitoring and quick change of the different physical parameters.

\section{Experimental setup}

The setup shown in Fig. 1 consists of a closed channel, with a racetrack shape, of about $3.6 \mathrm{~m}$ long and $W=100$ $\mathrm{mm}$ wide, composed of two linear parts of $900 \mathrm{~mm}$ long each separated by two opposite curved parts of radius 300 $\mathrm{mm}$. Water flow (density $\rho=1000 \mathrm{~kg} \mathrm{~m}^{-3}$ and height $h=160 \mathrm{~mm}$ ) is driven by means of a paddle wheel located in one linear part of the channel and is considered after a honeycomb in the other linear test section where a granular bed composed of glass beads of density $\rho_{g}=2500 \mathrm{~kg}$ $\mathrm{m}^{-3}$ and mean diameter $d=(270 \pm 30) \mu \mathrm{m}$ is put in a drawer of length $600 \mathrm{~mm}$ and $40 \mathrm{~mm}$ depth at the bottom of the channel. A vertical cylinder of diameter $D=20$ mm exceeds the granular bed from a height $H=90 \mathrm{~mm}$, lower that the water height. This cylinder is fixed at the bottom of the granular bed at the $2 / 3$ of the test section that we will take at the origin of the horizontal frame of reference $(x, y)$, with $x$ the streamwise direction and $y$ the

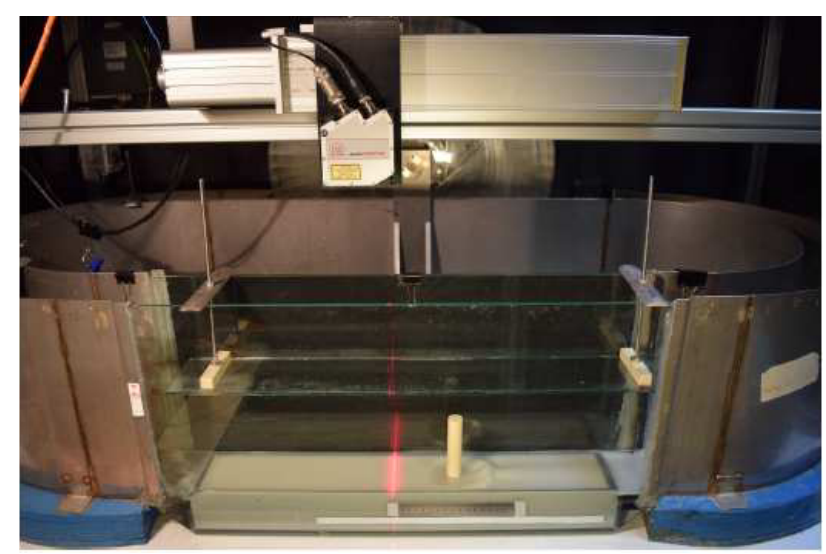

Figure 1. Picture of the experimental setup.

\footnotetext{
^e-mail: philippe.gondret@u-psud.fr
} 

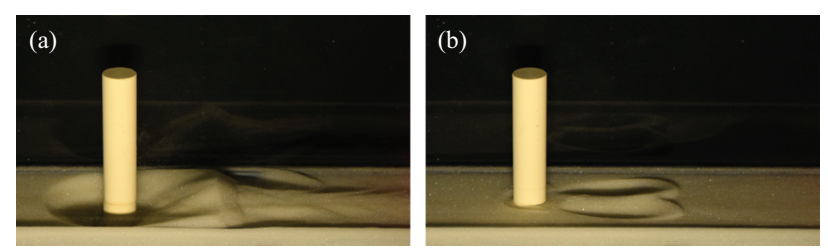

Figure 2. Snapshots of two different erosion patterns close to a cylinder: (a) "Horseshoe" pattern at the cylinder foot for $R e_{D} \simeq 3.610^{3}, S h \simeq 8$ and $t \simeq 610^{2} \mathrm{~s}$; (b) "Bunny ears" pattern downstream the cylinder for $R e_{D} \simeq 2.310^{3}, S h \simeq 3.3$ and $t \simeq 4.7610^{4} \mathrm{~s}$.

transverse direction. The side walls of the test section are made of glass to allow direct visualization of the scouring close to the cylinder. A laser profilometer is mounted on a horizontal translation guide in order to scan the granular bed topography $\xi(x, y)$ from above. To limit signal noise in the $\xi$ measurements that may arise from the fluctuations of the water surface, a horizontal glass wall is put at the water surface above the granular bed.

The flow velocity $U$ is controlled by the paddle wheel rotation in the typical range $0.05 \lesssim U \lesssim 0.25 \mathrm{~m} \mathrm{~s}^{-1}$ leading to the following typical Reynolds number $R e_{l}=U l / v$ for the water flow, depending on a length scale $l$ and the kinetic viscosity of water $v=10^{-6} \mathrm{~m}^{2} \mathrm{~s}^{-1}$. The Reynolds number based on the channel width $W$ is $R e_{W} \simeq 10^{4}$, corresponding thus to a turbulent regime. The Reynolds number of the flow around the cylinder of diameter $D$ is $R e_{D} \simeq 10^{3}$, far beyond the critical value 69 for the Bénard-von Kármán unsteadiness for an infinite cylinder between two parallel sidewalls with the present blockage ratio $D / W=0.2[6]$. The flow around the cylinder is thus also turbulent with strong unsteady wake vortices. The Reynolds number of the flow around the grains is $R e_{d} \simeq 50$, corresponding rather to a laminar regime. The Froude number $F r=U /(g h)^{1 / 2}$, defined as the ratio of the flow velocity to the velocity of surface waves, is smaller than 0.2 which thus corresponds to subcritical flows. Before any experiment, the granular bed is stirred and then leveled with a ruler for an initial flat horizontal surface. The cylinder is then fixed on its support without disrupting the granular bed, the water flow is turned on at a given velocity $U$ and the laser profilometer is turned on to measure the instantaneous granular bed topography $\xi(x, y, t)$ at regular time intervals $\Delta t$ adapted to the erosion dynamics without any flow stop. The instantaneous topography $\xi(x, y, t)$ is the difference from the initial topography $\xi(x, y, 0)$ before any flow.

\section{Experimental results}

Without any cylinder, erosion takes place on the granular bed above the critical value $S h_{0} \simeq 9$ for the global Shields number $S h=\rho U^{2} / \Delta \rho g d$ based here on the flow velocity $U$ far above the granular bed. The present Shields number is an inertial Shields number chosen as $R e_{d}>1$, which is different from the local Shields number that could be taken by knowing the exact fluid stress at the bed surface $[7,8]$.
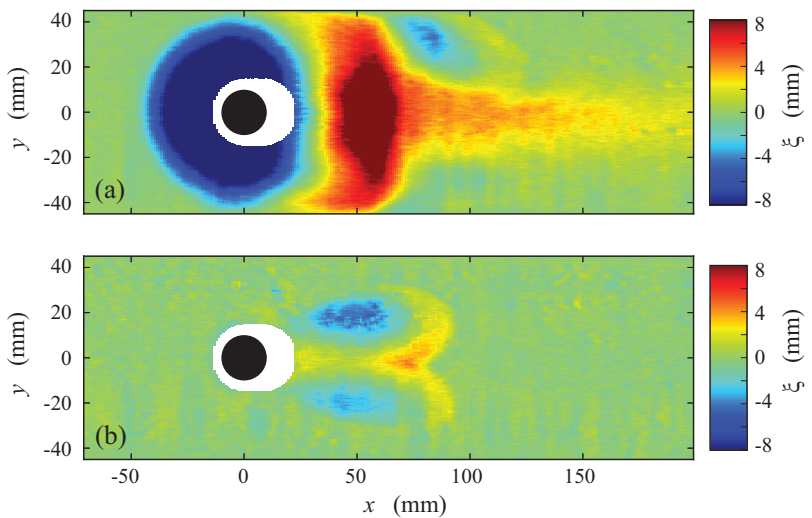

Figure 3. Topography $\xi(x, y)$ of the two different erosion patterns: (a) "Horseshoe" pattern at the cylinder foot for $R e_{D} \simeq$ $3.610^{3}, S h \simeq 8$ and $t \simeq 610^{2} \mathrm{~s}$; (b) "Bunny ears" pattern downstream the cylinder for $R e_{D} \simeq 2.310^{3}, S h \simeq 3.3$ and $t \simeq 4.7610^{4}$ s. Dark circles are the top view of the cylinder. White regions are zones that are not detected by the laser profilometer.

We work here in clear-water conditions $\left(S h<S h_{0}\right)$ for which no erosion occurs far from the cylinder. The presence of the cylinder disrupts the flow and creates several vortices [2]: a horseshoe vortex at the foot of the cylinder and wake vortices downstream. The erosion threshold of the granular bed is thus lowered due to the presence of these vortices which lead to scouring in the vicinity of the obstacle.

The different patterns observed are reported by imaging from the side and by laser profilometry in figures 2 and 3 respectively. At large enough flow velocity, but small enough to remain in clear-water conditions $(4 \lesssim S h \lesssim 10)$, one observes the classical erosion pattern with a deep scour hole at the foot of the cylinder due to the horseshoe vortex [Figs. 2(a) and 3(a)]. This erosion pattern arises in a short time scale of typically a few minutes in the present setup. At lower flow velocities $(1 \lesssim S h \lesssim 4)$, one observes an other erosion pattern with two elongated holes located a few diameters downstream the cylinder in an amazing "bunny ears" shape [Figs. 2(b) and 3(b)]. This erosion pattern arises typically in a few hours thus with a much larger time scale than the horseshoe pattern.

From the precise topography evolution $\xi(x, y, t)$ of the granular bed, one can extract the time evolution of the maximum scour depth $\delta(t)$ as shown in figures 4(a) and 4(b) for each erosion pattern respectively. In the case of the classical Horseshoe Scour (HSS), $\delta(t)$ increases quickly and tends to reach an almost constant asymptotic value $\delta_{\infty} \simeq D$ of the order of the cylinder diameter, which is in agreement with the usually reported values [3]. Note that the curves in Fig. 4(a) are truncated as soon as the pit reaches the side walls of the channel. By contrast, $\delta(t)$ increases much more slowly for the Bunny Ears Scour (BES) and reaches a much smaller asymptotic value $\delta_{\infty} \simeq 0.1 D$ than in the HSS case. Note that the position $\left(x_{m}, y_{m}\right)$ of the maximum scour depth does not evolves with time during the time depth evolution: HSS remains close to the upstream cylinder foot 

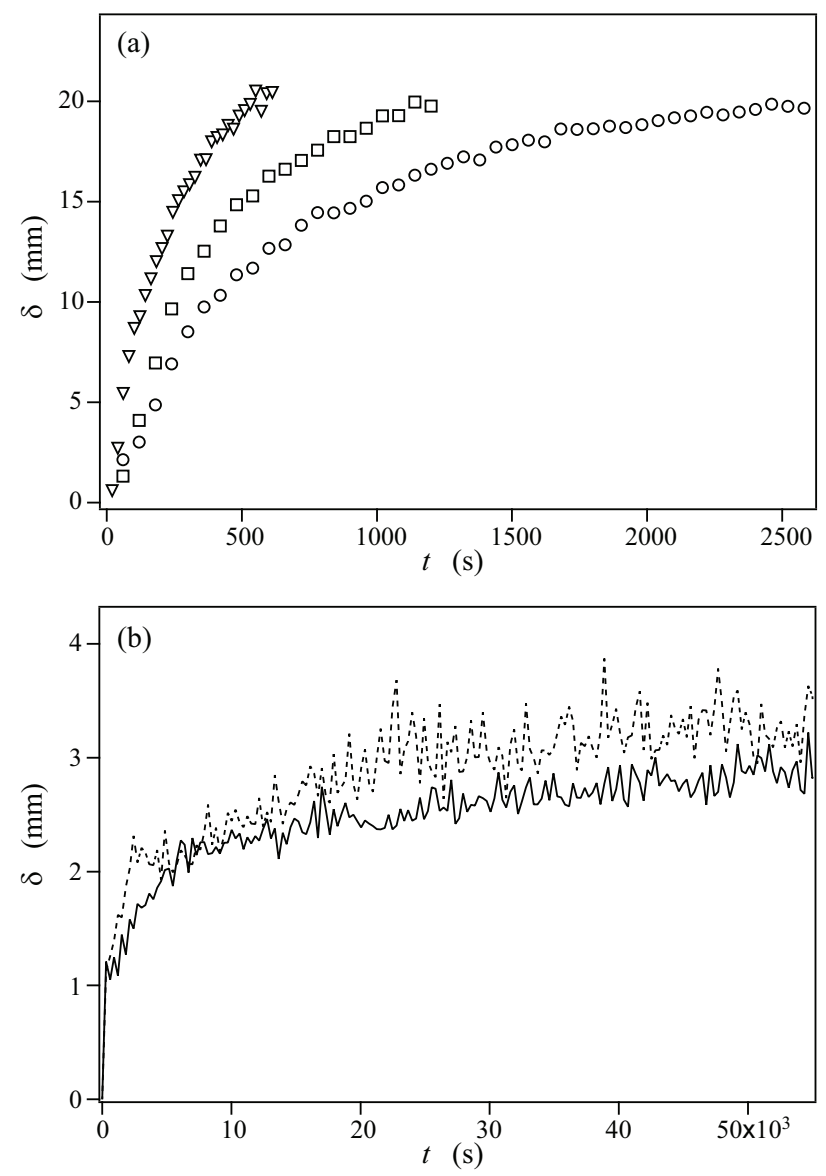

Figure 4. Time evolution of the depth $\delta$ for the two erosion patterns at different Reynolds numbers and different Shields numbers. (a) Horseshoe scour at (o) $R e_{D} \simeq 2.810^{3}, S h \simeq 5.1$, () $R e_{D} \simeq 3.210^{3}, S h \simeq 6.4$, and $(\nabla) R e_{D} \simeq 3.610^{3}, S h \simeq 8.0$. (b) Bunny ears scour at $(-) R e_{D} \simeq 2.110^{3}, S h \simeq 2.8$ and $(--) R e_{D} \simeq 2.310^{3}, S h \simeq 3.3$.

with $\left(x_{m}, y_{m}\right) \simeq(-D / 2,0)$ whereas BES is a few diameter downstream with $\left(x_{m}, y_{m}\right) \simeq(2 D, \pm D)$.

The evolution of the depth and position of these patterns as a function of the Reynolds number $R e_{D}$ or the Shields number $S h$ will be discussed. The critical Shields number above which HSS appears is larger than the critical Shields number for BES to appear. Note that BES is still observed for large Shields numbers but disturbed by the quicker and stronger HSS development. To investigate the far development of BES, a rigid sabot at the foot of the cylinder will be used. In that case, the HSS development is inhibited as no more granular bed can be eroded by the horseshoe vortex close to the cylinder.

\section{Conclusion}

With a small scale laboratory setup, the erosion in the vicinity of a vertical cylinder posed on a granular bed is investigated when submitted to a steady flow in clear-water conditions. Besides the well-known scour pattern that may arise quickly at the cylinder foot due to the horseshoe vortex, one observes an other erosion pattern downstream the cylinder with an amazing "bunny ears" shape, that appears much more slowly for weaker velocities. The conditions for this new pattern to appear must be refined together with its relation to the flow structure with the wakes vortices. The effects of the various vortices will be decoupled in our channel by replacing part of the granular bed by a nonerodible rigid floor. Additional experiments with Particle Image Velocimetry means are ongoing to investigate the complex velocity field and its detailed interaction with the granular bed.

\section{Acknowledgements}

This work is supported by a public grant of the French National Research Agency (ANR) within the project SSHEAR (grant No. ANR-14-CE03-001). We are grateful to S. Chateau for preliminary experiments and to E. Wesfreid for fruitful discussions. We thank J. Amarni, A. Aubertin, L. Auffray, and R. Pidoux for their contribution to the development of the experimental setup.

\section{References}

[1] A. Andreotti, Y. Forterre, O. Pouliquen, Granular Media - Between Fluid and Solid (Cambridge University Press, 2013)

[2] R. Ettema, G. Constantinescu, B. Melville, Evaluation of bridge scour research: Pier scour processes and predictions. Washington, DC: Transportation Research Board of the National Academies (2011)

[3] C. Manes, M. Brocchini, J. Fluid Mech 779, 309-324 (2015).

[4] C.H.K. Williamson, Ann. Rev. Fluid Mech. 28, 477 (1996)

[5] S. Kuang, C. LaMarche, J. Curtis, A. Yu, Powder Technol. 239, 319 (2013).

[6] J.-H. Chen, W.G. Pritchard, S.J. Tavener, J. Fluid Mech. 284, 23 (1995)

[7] S. Badr, G. Gauthier, P. Gondret, Phys. Fluids. 28, 033305 (2016)

[8] T. Loiseleux, P. Gondret, M. Rabaud, D. Doppler, Phys. Fluids 17, 103304 (2005) 